\section{Atividade física e escore de risco de Framingham entre idosos: Projeto Bambuí}

\author{
Physical activity and Framingham risk score in older \\ adults: the Bambui Health and Aging Study
}

\author{
Actividad física y la escala de riesgo de Framingham \\ entre ancianos: Proyecto Bambuí
}

This study explored the association between energy expenditure during physical activity and the Framingham risk score in the elderly. This was a cross-sectional study with a sample of 1,473 older adults. The dependent variable was level of physical activity, estimated by the metabolic equivalents in tertiles. The exploratory variables were the components of the Framingham risk score and the Framingham risk score itself. The force of associations was estimated by odds ratios and confidence intervals using ordinal logistic regression. Higher physical activity was associated with lower Framingham risk score, younger age, lower proportion of diabetes, and higher mean HDL levels. Higher energy expenditure was also associated with lower odds of smoking in men. The findings suggest that the adoption of a physically active lifestyle can reduce coronary risk, and that the promotion of physical activity is a universal strategy to fight chronic noncommunicable diseases.

Energy Expenditure; Coronary Disease; Aged; Exercise; Risk Factors
Jaqueline de Oliveira Santana 1,2 Juciany Rodrigues de Oliveira Ramalho 1 Josélia Oliveira Araújo Firmo 1 Maria Fernanda Lima-Costa 1 Sérgio Viana Peixoto ${ }^{3}$

\section{Resumo}

O trabalho explorou a associação entre o gasto energético em atividades físicas e o escore de risco de Framingham entre idosos. Trata-se de um estudo seccional, incluindo 1.473 idosos. A variável dependente foi o nível de atividade física, estimado pela taxa de equivalentes metabólicos, em tercis. As variáveis exploratórias foram os componentes do escore de risco de Framingham e o próprio escore de risco de Framingham. A estimativa da força das associações foi baseada no cálculo do odds ratio e intervalos de confiança, utilizando a regressão logística ordinal. O maior nível de atividade física esteve associado a menores valores do escore de risco de Framingham, menor idade, menor proporção de diabetes e maiores valores médios de HDL. O maior gasto energético esteve ainda associado à menor chance de ser fumante entre os homens. Sugere-se que a adoção de um estilo de vida fisicamente ativo pode reduzir o risco coronariano, sendo o incentivo à prática da atividade física uma estratégia universal de combate às doenças crônicas não transmissiveis.

Gasto Energético; Doença das Coronárias; Idoso; Exercício; Fatores de Risco 


\section{Introdução}

A inatividade física está associada à elevada carga de doenças crônicas não transmissíveis (DCNT) 1 , sendo que a prática de atividade física parece alterar favoravelmente alguns fatores de risco para doença coronariana, como tabagismo, hipertensão, hiperlipidemias e diabetes, embora os resultados apresentem variações importantes entre diferentes populações 2,3 . O rápido envelhecimento populacional e o consequente aumento das DCNT, como observado no Brasil, justificam estudos sobre a prática da atividade física e seus fatores associados nesse grupo etário 4 .

$\mathrm{O}$ risco coronariano pode ser avaliado de diversas maneiras, sendo o escore de risco de Framingham 5 o mais utilizado 6 . No entanto, a associação entre a prática de atividade física e o escore de risco de Framingham é pouco explorada na literatura, sobretudo em populações idosas e naquelas residentes em países de baixa e média renda. Estudos em populações adultas da Finlândia e Inglaterra demonstraram que a inatividade física foi associada ao maior risco coronariano entre homens e mulheres e em diferentes categorias do escore de risco de Framingham 7,8. Além disso, a atividade física aumentou a capacidade de predição do escore de risco de Framingham em estudo longitudinal entre americanos adultos 9 .

Nesse contexto, explorou-se a associação entre o gasto energético em atividade física e o escore de risco de Framingham (e seus componentes), segundo sexo, entre idosos brasileiros residentes na comunidade.

\section{Métodos}

Trata-se de um estudo transversal, incluindo 1.473 indivíduos com 60 anos ou mais $(84,6 \%$ dos residentes idosos), participantes da linha de base da Coorte de Idosos de Bambuí, estabelecida em 1997 e conduzida na cidade de Bambuí, Minas Gerais, Brasil 10.

A variável dependente foi o gasto energético em atividade física, estimado pelo cálculo da taxa de equivalentes metabólicos (MET-minuto/ semana), utilizando-se um questionário sobre a prática de atividade física global (lazer, trabalho, deslocamento, doméstica) nos últimos 90 dias, com informações sobre duração, frequência semanal e tipo de atividade, e considerando apenas as atividades realizadas por 10 minutos ou mais ${ }^{11}$. O gasto energético foi dividido em tercis para cada sexo, caracterizando a população nos níveis de atividade física: baixo (primeiro tercil), médio (segundo tercil) e alto (terceiro tercil).
O escore de risco de Framingham foi calculado considerando-se as variáveis idade, sexo, colesterol total, HDL, pressão arterial sistólica e diastólica, tabagismo e diabetes, conforme estabelecido na literatura 5 , tendo sido avaliado como variável contínua.

As entrevistas foram realizadas na residência do participante e respondidas pelo próprio idoso ou por um respondente próximo $(4,8 \%)$, e a coleta de sangue (recomendação de jejum de 12 horas) e exames físicos foram realizados na clínica de campo do projeto. A coleta de dados foi realizada por pessoal treinado, utilizando procedimentos e instrumentos padronizados 10 .

Tabagistas atuais foram definidos como aqueles que fumaram no mínimo 100 cigarros durante a vida e continuavam fumando no momento da entrevista. Diabetes foi definido como glicemia de jejum > 140mg/dL e/ou uso de insulina ou hipoglicemiantes orais, conforme proposta de construção do escore de risco de Framingham 5. O uso de medicamentos foi aferido pela verificação da embalagem ou da prescrição médica no momento da visita domiciliar.

Para comparação das variáveis estudadas e os tercis de gasto energético utilizou-se o teste do qui-quadrado de Pearson e a análise de variância para comparações entre proporções e médias, respectivamente. A associação entre gasto energético e escore de risco de Framingham, e seus componentes, foi estimada pela regressão logística ordinal (modelo de odds proporcionais). A suposição de proporcionalidade foi avaliada pelo teste da razão de verossimilhança $(p>0,05)$. As análises foram estratificadas por sexo e os modelos ajustados por idade, com exceção do escore de risco de Framingham, que já considera esta variável, utilizando-se o Stata 13.0 (StataCorp LP, College Station, Estados Unidos).

\section{Resultados}

A média de idade foi igual a 69,1 anos (desvio padrão $=7,2$ anos), não diferindo entre os sexos ( $\mathrm{p}=$ 0,174 ). As Tabelas 1 e 2 mostram a distribuição das variáveis consideradas no estudo, segundo categorias do gasto energético, entre homens e mulheres, respectivamente.

A Tabela 3 mostra a associação entre as variáveis de risco coronariano e os níveis de gasto energético. Em ambos os sexos, maior nível de atividade física esteve significativamente associado a menores valores médios do escore de risco de Framingham, menor idade, menor proporção de diabetes e maiores valores médios de HDL. Ademais, o maior gasto energético esteve 
Perfil de risco coronariano entre homens idosos, segundo o nível de atividade física. Linha de base da Coorte de Idosos de Bambuí, Minas Gerais, Brasil.

\begin{tabular}{|c|c|c|c|c|c|}
\hline \multirow[t]{2}{*}{ Variáveis } & \multirow{2}{*}{$\begin{array}{c}\text { Total } \\
(\mathrm{N}=578)\end{array}$} & \multicolumn{3}{|c|}{ Gasto energético } & \multirow[t]{2}{*}{ Valor de p * } \\
\hline & & $\begin{array}{c}\text { Baixo } \\
(n=185)\end{array}$ & $\begin{array}{l}\text { Médio } \\
(n=196)\end{array}$ & $\begin{array}{c}\text { Alto } \\
(n=197)\end{array}$ & \\
\hline Escore de risco em \%, média (DP) & $9,5(2,5)$ & $9,9(2,5)$ & $9,6(2,5)$ & $8,9(2,3)$ & $<0,001$ \\
\hline Idade em anos, média (DP) & $68,8(7,1)$ & $70,7(7,7)$ & $68,9(7,2)$ & $66,9(7,1)$ & $<0,001$ \\
\hline Tabagismo atual (\%) & 30,3 & 39,5 & 28,6 & 23,4 & 0,002 \\
\hline Diabetes [tratamento ou glicemia de jejum > 140g/dL] (\%) & 9,2 & 11,9 & 11,7 & 4,1 & 0,009 \\
\hline Pressão arterial sistólica em mmHg, média (DP) & $137,7(22,9)$ & $138,9(27,0)$ & $139,5(21,2)$ & $134,9(20,1)$ & 0,094 \\
\hline Pressão arterial diastólica em mmHg, média (DP) & $85,1(13,2)$ & $85,1(15,3)$ & $85,9(12,6)$ & $84,3(11,5)$ & 0,489 \\
\hline Colesterol HDL em mg/dL, média (DP) & $46,8(15,3)$ & $45,2(15,4)$ & $46,5(15,8)$ & $48,5(14,7)$ & 0,103 \\
\hline Colesterol total em mg/dL, média (DP) & $220,3(46,1)$ & $216,1(46,8)$ & $219,63(44,8)$ & $225,0(46,4)$ & 0,160 \\
\hline
\end{tabular}

DP: desvio padrão.

* Valor de p para o teste do qui-quadrado de Pearson ou análise de variância.

Tabela 2

Perfil de risco coronariano entre mulheres idosas, segundo o nível de atividade física. Linha de base da Coorte de Idosos de Bambuí, Minas Gerais, Brasil.

\begin{tabular}{|c|c|c|c|c|c|}
\hline \multirow[t]{2}{*}{ Variáveis } & \multirow{2}{*}{$\begin{array}{c}\text { Total } \\
(\mathrm{N}=895)\end{array}$} & \multicolumn{3}{|c|}{ Gasto energético } & \multirow[t]{2}{*}{ Valor de $p$ * } \\
\hline & & $\begin{array}{c}\text { Baixo } \\
(n=286)\end{array}$ & $\begin{array}{c}\text { Médio } \\
(n=300)\end{array}$ & $\begin{array}{c}\text { Alto } \\
(n=309)\end{array}$ & \\
\hline Escore de risco em \%, média (DP) & $10,8(3,8)$ & $11,0(4,1)$ & $11,1(3,7)$ & $10,3(3,6)$ & 0,024 \\
\hline Idade em anos, média (DP) & $69,3(7,2)$ & $71,9(8,0)$ & $68,9(6,8)$ & $67,3(6,2)$ & $<0,001$ \\
\hline Tabagismo atual (\%) & 10,2 & 9,1 & 10,7 & 10,7 & 0,766 \\
\hline Diabetes [tratamento ou glicemia de jejum > 140g/dL] (\%) & 10,8 & 14,0 & 10,7 & 8,1 & 0,069 \\
\hline Pressão arterial sistólica em mmHg, média (DP) & $137,2(22,4)$ & $139,0(23,9)$ & $137,4(22,3)$ & $135,2(21,0)$ & 0,109 \\
\hline Pressão arterial diastólica em mmHg, média (DP) & $82,3(12,2)$ & $81,6(12,9)$ & $83,7(12,4)$ & $81,7(11,2)$ & 0,059 \\
\hline Colesterol HDL em mg/dL, média (DP) & $50,7(14,6)$ & $49,9(15,6)$ & $50,5(14,0)$ & $51,6(14,3)$ & 0,371 \\
\hline Colesterol total em mg/dL, média (DP) & $240,9(49,1)$ & $235,2(49,3)$ & $244,8(48,2)$ & $242,5(49,5)$ & 0,049 \\
\hline
\end{tabular}

DP: desvio padrão.

* Valor de p para o teste do qui-quadrado de Pearson ou análise de variância.

associado à menor chance de ser fumante entre os homens.

\section{Discussão}

O principal resultado deste trabalho refere-se ao efeito benéfico da atividade física no risco coronariano de idosos residentes em um país latinoamericano. O maior gasto energético foi também mais comum entre os idosos mais jovens, não fumantes (para homens), não diabéticos e com maiores valores de HDL.
Estudos mostram a importância da prática da atividade física, considerando sua intensidade, sendo que maior gasto energético tem sido associado à maior redução do risco coronariano 12,13, reforçando a importância dessa prática para prevenção de eventos coronarianos, podendo ainda aumentar a capacidade preditiva do escore de risco de Framingham 8,9. Entre os idosos residentes em Bambuí, a atividade física foi consistentemente associada ao menor risco para doenças do coração, em ambos os sexos, confirmando os resultados observados em países mais ricos 8,12. 
Associação entre o escore de risco de Framingham e seus componentes com o gasto energético (em tercis) entre idosos, segundo sexo. Linha de base da Coorte de Idosos de Bambuí, Minas Gerais, Brasil.

\begin{tabular}{|c|c|c|}
\hline \multirow[t]{2}{*}{ Variáveis } & \multicolumn{2}{|c|}{ OR (IC95\%) } \\
\hline & Homens & Mulheres \\
\hline Escore de risco (\%) & $0,88(0,83-0,94) *$ & $0,96(0,93-0,99)$ * \\
\hline Idade (anos) & $0,95(0,92-0,97)$ * & $0,93(0,92-0,95)$ * \\
\hline \multicolumn{3}{|l|}{ Tabagismo atual } \\
\hline Não & 1,00 & 1,00 \\
\hline Sim & $0,53(0,38-0,74) *$ & $0,99(0,66-1,48)$ \\
\hline \multicolumn{3}{|c|}{ Diabetes (tratamento ou glicemia de jejum > 140g/dL) } \\
\hline Não & 1,00 & 1,00 \\
\hline Sim & $0,48(0,28-0,80)$ * & $0,64(0,43-0,96)$ * \\
\hline Pressão arterial sistólica (mmHg) & $0,99(0,98-1,01)$ & $1,00(0,99-1,01)$ \\
\hline Pressão arterial diastólica (mmHg) & $0,99(0,98-1,01)$ & $1,00(0,99-1,01)$ \\
\hline Colesterol HDL (mg/dL) & $1,01(1,01-1,02)$ * & $1,01(1,00-1,02)$ * \\
\hline Colesterol total (mg/dL) & $1,00(1,00-1,01)$ & $1,00(1,00-1,01)$ \\
\hline
\end{tabular}

IC95\%: intervalo de 95\% de confiança; OR: odds ratio.

Nota: OR e IC95\% obtidos pela regressão logística ordinal, considerando o gasto energético em atividades físicas dividido em tercis, para cada sexo; valores ajustados por idade, com exceção do escore de risco.

${ }^{*} \mathrm{p}<0,05$.

O aumento da idade é associado a menores valores de gasto energético, conforme observado em outras populações 14,15, chamando atenção para a necessidade do incentivo à prática de atividade física também nesse grupo. Conforme observado em Bambuí, evidências mostram a eficácia da atividade física na prevenção e tratamento do diabetes 14 , ressaltando que a melhora na sensibilidade à insulina é influenciada também pela intensidade do exercício 16 . $\mathrm{Na}$ análise realizada com o ponto de corte atualmente recomendado para o diagnóstico de diabetes (tratamento e/ ou glicemia de jejum $\geq 126 \mathrm{mg} / \mathrm{dL}$ ), a associação manteve-se na mesma direção.

Apesar de a atividade física estar associada a um perfil lipídico favorável em algumas populações 15, como verificado em Bambuí para os níveis de HDL, alguns estudos não observaram esta associação 3,17. Em relação ao hábito de fumar, os resultados corroboram a literatura 18 , sugerindo uma convergência de comportamentos saudá- veis também em idosos. A não associação observada entre as mulheres pode estar relacionada ao baixo percentual de tabagistas atuais $(10,2 \%)$ nesta população.

Entre as limitações do trabalho, destaca-se a impossibilidade de se estabelecer relação temporal entre as variáveis e a utilização de informação autorreferida para avaliar o gasto energético. Por outro lado, é um estudo de base populacional, usando protocolos padronizados e com rigoroso controle de qualidade, com avaliação detalhada da atividade física, permitindo a estimativa do gasto energético global.

Portanto, os resultados demonstram o possível efeito da prática de atividade física na diminuição do risco coronariano entre idosos brasileiros de ambos os sexos, corroborando os resultados observados em outras populações, o que fala a favor do incentivo a essa prática como uma estratégia universal de combate às DCNT, mesmo na população idosa. 


\section{Resumen}

Este estudio exploró la asociación entre el gasto de energía en actividades físicas y la escala de riesgo de Framingham entre ancianos. Se trata de un estudio transversal, incluyendo 1.473 ancianos. La variable dependiente fue el nivel de actrividade física, estimado por la tasa de equivalentes metabólicos, en terciles. Las variables explicativas fueron los componentes de la escala de riesgo de Framingham y el propio escala de riesgo de Framingham. La estimación de la fuerza de las asociaciones se basa en el cálculo de odds ratios y los intervalos de confianza, mediante regresión logística ordinal. El nivel más alto de actividade física se asoció con los valores más bajos de la escala de riesgo de Framingham, la edad más joven, menor proporción de diabetes y el aumento de HDL promedio. El nivel más alto de gasto de energía se asoció con menor probabilidad de ser un fumador entre los hombres. Se sugiere que la adopción de un estilo de vida físicamente activo puede reducir el riesgo coronario. El fomento de la práctica de actividad física es una estrategia universal para combatir las enfermedades crónicas.

Gasto de Energía; Enfermedad Coronaria; Anciano; Ejercicio; Factores de Riesgo

\section{Colaboradores}

J. O. Santana e J. R. O. Ramalho participaram da análise dos dados, interpretação dos resultados e elaboração do artigo. J. O. A. Firmo e M. F. Lima-Costa contribuíram para o desenho do estudo e análise crítica do artigo. S. V. Peixoto participou da análise e interpretação dos dados, elaboração e aprovação da versão final do artigo.

\section{Agradecimentos}

Os autores agradecem à Financiadora de Estudos e Projetos (FINEP), ao Centro de Pesquisas René Rachou, Fundação Oswaldo Cruz e ao Conselho Nacional de Desenvolvimento Científico e Tecnológico (CNPq) pelo financiamento do estudo. J. O. A. Firmo, M. F. Lima-Costa e S. V. Peixoto são bolsistas de produtividade do CNPq.

\section{Referências}

1. Kohl HW, Craig CL, Lambert EV, Inoue S, Alkandar JR, Leetongin G, et al. The pandemic of physical inactivity: global action for public health. Lancet 2012; 380:294-305.

2. Batty GD. Physical activity and coronary heart disease in older adults. Eur J Public Health 2002; 12:171-6.

3. Cox KL, Burke V, Beilin LJ, Puddey IB. A comparison of the effects of swimming and walking on body weight, fat distribution, lipids, glucose, and insulin in older women: the Sedentary Women Exercise Adherence Trial 2. Metabolism 2010; 59:1562-73.

4. American College of Sports Medicine; ChodzkoZajko WJ, Proctor DN, Fiatarone Singh MA, Minson CT, Nigg CR, et al. Exercise and physical activity for older adults. Med Sci Sports Exerc 2009; 41: 1510-30.
5. Wilson PW, D'Agostino RB, Levy D, Belanger AD, Silbershatz H, Kannel WB. Prediction of coronary heart disease using risk factor categories. Circulation 1998; 97:1837-47.

6. Rodondi N, Locatelli I, Aujesky D, Butler J, Vittinghoff E, Simonsick E, et al. Framingham risk score and alternatives for prediction of coronary heart disease in older adults. PLoS One 2012; 7:e34287.

7. Hu G, Tuomilehto J, Borodulin K, Jousilahti P. The joint associations of occupational, commuting, and leisure-time physical activity, and Framingham risk score on the 10-year risk of coronary heart disease. Eur Heart J 2007; 28:492-8.

8. Arsenault BJ, Rana JS, Lemieux I, Després JP, Wareham NJ, Kastelein JJ, et al. Physical activity, the Framingham risk score and risk of coronary heart disease in men and women of the EPIC-Norfolk study. Atherosclerosis 2010; 209:261-5. 
9. Sacco RL, Khatri M, Rundek T, Xu Q, Gardener H, Boden-Albala B, et al. Improving global vascular risk prediction with behavioral and anthropometric factors. The multiethnic NOMAS (Northern Manhattan Cohort Study). J Am Coll Cardiol 2009; 54:2303-11.

10. Lima-Costa MF, Firmo JOA, Uchoa E. The Bambuí Cohort Study of Aging: methodology and health profile of participants at baseline. Cad Saúde Pública 2011; 27 Suppl 3:S327-35.

11. Ramalho JRO, Lima-Costa MF, Firmo JOA, Peixoto SV. Energy expenditure through physical activity in a population of community-dwelling Brazilian elderly: cross-sectional evidences from the Bambuí cohort study of aging. Cad Saúde Pública 2011; 27 Suppl 3:S399-408

12. Yu S, Yarnell JW, Sweetnam PM, Murray L. What level of physical activity protects against premature cardiovascular death? The Caerphilly Study. Heart 2003; 89:502-6.

13. Oguma Y, Shinoda-Tagawa T. Physical activity decreases cardiovascular disease risk in women: review and meta-analysis. Am J Prev Med 2004; 26:407-18.
14. Demakokos P, Hamer M, Stamatakis E, Steptoe A Low-intensity physical activity is associated with reduced risk of incident type 2 diabetes in older adults: evidence from the English Longitudinal Study of Ageing. Diabetologia 2010; 53:1877-85.

15. Panagiotakos DB, Pitsavos C, Chrysohoou C, Skoumas J, Zeimbekis A, Papaioannou I, et al. Effect of leisure time physical activity on blood lipid levels: the ATTICA study. Coron Artery Dis 2003; 14:533-9.

16. Segerström AB, Glans F, Eriksson KF, Holmbäck AM, Groop L, Thorsson O, et al. Impact of exercise intensity and duration on insulin sensitivity in women with T2D. Eur J Intern Med 2010; 21:404-8.

17. Sillanpää E, Laaksonen DE, Häkkinen A, Karavirta L, Jensen B, Kraemer WJ, et al. Body composition, fitness, and metabolic health during strength and endurance training and their combination in middle-aged and older women. Eur J Appl Physiol 2009; 106:285-96.

18. Zaitune MPA, Barros MBA, Lima MG, César CLG, Carandina L, Goldbaum M, et al. Fatores associados ao tabagismo em idosos: inquérito de saúde no Estado de São Paulo (ISA-SP). Cad Saúde Pública 2012; 28:583-96.

Recebido em 10/Abr/2015

Versão final reapresentada em 10/Jul/2015

Aprovado em 27/Ago/2015 